\title{
Percepção do enfermeiro sobre promoção da saúde na Unidade de Terapia Intensiva"
}

\author{
THE NURSES' PERCEPTIONS REGARDING HEALTH PROMOTION \\ IN THE INTENSIVE CARE UNIT \\ PERCEPCIÓN DEL ENFERMERO SOBRE PROMOCIÓN DE LA SALUD \\ EN LA UNIDAD DE TERAPIA INTENSIVA
}

\section{Adriana Sousa Carvalho de Aguiar ${ }^{1}$, Monaliza Ribeiro Mariano ${ }^{2}$, Lívia Silva Almeida ${ }^{3}$, Maria Vera Lúcia Moreira Leitão Cardoso ${ }^{4}$, Lorita Marlena Freitag Pagliuca ${ }^{5}$, Cristiana Brasil de Almeida Rebouças ${ }^{6}$}

\section{RESUMO}

Objetivou-se relatar a percepção dos enfermeiros sobre a promoção da saúde, descrever ações de promoção da saúde e identificar dificuldades na realização de atividades de promoção da saúde na Unidade de Terapia Intensiva (UTI). Trata-se de um estudo descritivo, exploratório, qualitativo realizado com 31 enfermeiros de duas UTIs adulto e uma UTI neonatal de hospital de referência em Fortaleza, Ceará, Brasil, entre julho e agosto de 2009, mediante questionário. Os dados foram analisados e categorizados a partir da análise de conteúdo de Bardin. Emergiram as seguintes categorias e subcategorias: Conceito de promoção da saúde: visão biomédica $x$ visão holística de promoção da saúde; Ações de promoção da saúde; Comunicação e apoio emocional ao paciente/família; Promoção da saúde na UTI; Promoção da saúde com enfoque na educação em saúde; e Dificuldades para o desenvolvimento de ações de promoção da saúde.

\section{DESCRITORES}

Promoção da saúde

Unidades de Terapia Intensiva

Cuidados de enfermagem

\begin{abstract}
The objective of this study was to report the nurses' perceptions regarding health promotion, describe health promotion activities, and identify difficulties in performing health promotion activities in the Intensive Care Unit (ICU). This descriptive, exploratory study was performed with 31 nurses from two adult ICUs and one neonatal ICU of a reference hospital in Fortaleza, Ceará, Brazil, between July and August 2009 , using a questionnaire. Data were analyzed and categorized using Bardin's content analysis. The following categories and subcategories emerged: the concept of health promotion: biomedical and holistic view of health promotion; health promotion activities; communication and emotional support for patients/family; health promotion in the ICU; health promotion focusing on health education; and the difficulties in developing health promotion activities.
\end{abstract}

\section{DESCRIPTORS \\ Health promotion \\ Intensive Care Units \\ Nursing care}

\begin{abstract}
RESUMEN
Se objetivó relatar la percepción de enfermeros sobre promoción de la salud, describir acciones de promoción de salud e identificar dificultades en la realización de actividades de promoción de salud en Unidad de Terapia Intensiva (UTI). Estudio descriptivo, exploratorio, cualitativo, realizado con 31 enfermeros de dos UTIs de adultos y una UTI neonatal de hospital de referencia en Fortaleza-CE-Brasil, entre julio y agosto 2009, mediante cuestionario. Datos analizados y categorizados a partir de análisis de contenido de Bardin. Emergieron las siguientes categorías y subcategorías: Concepto de la promoción de la salud: visión biomédica $x$ visión holística de promoción de la salud; Acciones de promoción de la salud; Comunicación y apoyo emocional al paciente/familia; Promoción de la salud en la UTI; Promoción de la salud con enfoque en la educación sanitaria; y Dificultades para el desarrollo de acciones de promoción de la salud.
\end{abstract}

\author{
DESCRIPTORES \\ Promoción de la salud \\ Unidades de Terapia Intensiva \\ Atención de enfermería
}

\footnotetext{
* Extraído da disciplina "Bases Teóricas da Promoção da Saúde" do Curso de Pós-Graduação em Enfermagem da Universidade Federal do Ceará, 2010. 1 Enfermeira. Mestranda do Programa de Pós-Graduação em Enfermagem da Universidade Federal do Ceará. Bolsista FUNCAP. Fortaleza, CE, Brasil. adrianaufc@gmail.com ${ }^{2}$ Enfermeira. Mestranda do Programa de Pós-Graduação em Enfermagem da Universidade Federal do Ceará. Bolsista FUNCAP. Fortaleza, CE, Brasil. monalizamariano@yahoo.com.br ${ }^{3}$ Enfermeira. Mestranda do Programa de Pós-Graduação em Enfermagem da Universidade Federal do Ceará. Fortaleza, CE, Brasil. almeilivia@gmail.com ${ }^{4}$ Enfermeira. Doutora em Enfermagem. Pós-Doutorado em Vancouver/Canadá. Professora Associada do Programa de Pós-Graduação em Enfermagem da Universidade Federal do Ceará. Fortaleza, CE, Brasil. cardoso@ufc.br ${ }^{5}$ Enfermeira. Doutora em Enfermagem. Professora Titular do Programa de Pós-Graduação em Enfermagem da Universidade Federal do Ceará. Bolsista de Produtividade do CNPq. Fortaleza, CE, Brasil. pagliuca@ufc.br ${ }^{6}$ Enfermeira. Doutora em Enfermagem. Pós-Doutoranda em Enfermagem. Professora Colaboradora do Programa de Pós-Graduação em Enfermagem da Universidade Federal do Ceará. Bolsista CNPq. Fortaleza, CE, Brasil. cristianareboucas@yahoo.com.br
} 


\section{INTRODUÇÃO}

Nos últimos anos, a promoção da saúde tem se constituído um dos assuntos mais discutidos nos diferentes espaços da produção do conhecimento e das práticas de saúde. Mencionado tema tem permeado diversos cenários em âmbito nacional e internacional, corroborando para um conceito ampliado de saúde.

As ideias sobre promoção da saúde foram introduzidas no Brasil em meados dos anos de 1980, quando em debate a Reforma Sanitária. Além disso, influenciou também alguns movimentos como a VIII Conferência Nacional de Saúde; a Constituição de 1988; e a criação do Sistema Único de Saúde. Nos dias atuais, contribui ainda para a (re) estruturação da Estratégia Saúde da Família cujas bases representam o ideal de promoção da saúde ${ }^{(1)}$.

A ideia de promoção envolve o fortalecimento da capacidade individual e coletiva para lidar com a multiplicidade dos condicionantes da saúde. Portanto, promover saúde vai além da ausência de doença; deve ser entendido como uma estratégia transversal, multi e interdisciplinar. Diante desta concepção, não pode se limitar a questões relativas à prevenção, tratamento e cura de doenças $^{(2)}$. Abrange todas as ações direcionadas ao cuidado em si, independente do ambiente onde este cuidado é realizado.

Entretanto, é comum associar as estratégias de promoção da saúde ao contexto da saúde pública, pois nesse nível de atenção tais atividades mostram-se de forma mais evidente, em especial porque o foco principal é a família, ou o indivíduo inserido no ambiente onde vive. No ambiente hospitalar, onde os cuidados do enfermeiro estão mais direcionados para o aspecto curativo ou preventivo da doença, muitas vezes as ações de promoção da saúde tornam-se limitadas ou pouco valorizadas. Inserido nessa temática, nota-se escassez de investigação sobre como a promoção da saúde poderia ser incorporada, com sucesso, à atuação da enfermagem, e como os enfermeiros percebem seu papel ${ }^{(3)}$.

No tocante à Unidade de Terapia Intensiva (UTI), estudos nesse campo têm demonstrado que em decorrência da sua especificidade e grande diversidade tecnológica a assistência de enfermagem nestas unidades traz em seu escopo particularidades que a diferenciam das outras ${ }^{(4)}$. Referida unidade é dotada de pessoal qualificado e oferece uma assistência contínua com o uso de aparelhos sofisticados capazes de manter a sobrevida do paciente, exigindo dos seus profissionais alto nível de conhecimento, além de agilidade e atenção rigorosa na assistência prestada.

Em face dos aspectos inerentes ao contexto da UTI, estes podem contribuir para que ações de promoção da saúde no cuidado ao paciente tratado nessa unidade tornem-se pouco valorizados, desvinculados da prática pro- fissional ou até mesmo implícitos no ato de cuidar, passando, às vezes, despercebidos.

Com base nisso, as questões norteadoras da pesquisa foram: os enfermeiros compreendem a promoção da saúde no seu sentido mais abrangente? Eles desenvolvem ou não ações de promoção da saúde? Como as relacionam com o ato do cuidar? Tais ações estão restritas ao tratamento de doenças? Desse modo, o estudo pretende contribuir para a reflexão e a formação de uma prática profissional voltada para a promoção da saúde em seu sentido mais amplo.

Objetivou-se relatar a percepção dos enfermeiros sobre o significado de promoção da saúde; descrever as ações de promoção da saúde realizadas por enfermeiros no cuidado ao paciente tratado em unidade de terapia intensiva; e identificar as dificuldades apontadas por enfermeiros na realização de atividades de promoção da saúde na unidade de terapia intensiva.

\section{MÉTODO}

Estudo descritivo, exploratório, qualitativo realizado com enfermeiros de duas UTIs adultos e de uma UTI neonatal de um hospital público de referência em Fortaleza. Nas três unidades mencionadas trabalham 47 enfermeiros distribuídos nos turnos diurno e/ou noturno. A quantidade de sujeitos foi definida pela saturação dos dados, segundo a relevância dos conteúdos dos discursos pertinentes ao delineamento do objeto desta pesquisa. Foram excluídos do estudo enfermeiros que se encontravam de licença do trabalho ou de férias durante o período de coleta de dados.

Procedeu-se à coleta de dados nos meses de julho e agosto de 2009 e utilizou-se questionário semiestruturado, o qual foi respondido pelos enfermeiros das unidades citadas, sendo posteriormente recolhido pelas pesquisadoras. Tal instrumento foi constituído por duas partes: a primeira pontuou aspectos relacionados à caracterização dos profissionais e a segunda investigou o discurso do enfermeiro quanto à definição de promoção da saúde, atividades relacionadas a essa prática e dificuldades encontradas para sua implantação.

Após o preenchimento do instrumento, as informações foram analisadas segundo o método de análise de conteúdo(5). Os dados foram agrupados em categorias e subcategorias acerca da temática promoção da saúde. Para preservar o anonimato dos participantes, os discursos foram identificados pela letra $A$, seguida do número correspondente à sequência das entrevistas.

Como determinado, o projeto foi aprovado pelo Comitê de Ética da instituição com protocolo no 020702/09 e o Termo de Consentimento Livre e Esclarecido foi assi- 
nado pelos participantes do estudo, sendo-Ihes conferidos todos os direitos conforme os aspectos éticos para pesquisa com seres humanos.

\section{RESULTADOS E DISCUSSÃO}

Participaram da pesquisa 31 enfermeiros, dos quais 17 trabalhavam na UTI adulta e 14 na UTI neonatal. A maioria era do sexo feminino, com idade média de 32 anos e um tempo médio de oito anos de exercício na profissão de enfermeiro. Quanto à maior titulação, 16 deles possuíam o título de especialista, três possuíam mestrado e outros 12 graduados.

A partir do método de análise de conteúdo e de acordo com as informações obtidas por meio do preenchimento dos questionários pelos enfermeiros, foram delimitadas três categorias apresentadas e discutidas a seguir com as respectivas subcategorias: 1 . Conceito de promoção da saúde: visão biomédica $x$ visão holística da promoção da saúde; 2 . Ações de promoção da saúde na unidade de terapia intensiva; 3 . Dificuldades para o desenvolvimento de ações de promoção da saúde.

\section{Conceito de promoção da saúde: visão biomédica $x$ visão holística de promoção da saúde}

Nesta categoria buscou-se avaliar as concepções dos enfermeiros acerca do conceito de promoção da saúde e sua relação com o ambiente hospitalar, mais particularmente com a UTI. Da análise dos discursos, emergiram conceitos relacionados à visão biomédica e holística de promoção da saúde.

Observou-se, portanto, nas concepções de promoção da saúde relatadas por enfermeiros, conceitos que variavam de uma visão reduzida para uma visão ampliada de promoção da saúde. Percebeu-se entre os discursos, que estes entendem a promoção da saúde como o estabelecimento de medidas curativas e preventivas, focadas no processo de adoecimento. Contudo, sabe-se que a promoção da saúde é mais abrangente e transcende o enfoque estritamente biológico.

É o ato de proteger o paciente de alguma doença e/ou complicação (A13).

É a prevenção de doenças; realizar procedimentos para que se mantenha ou promova a saúde (A9).

(...) são práticas saudáveis com enfoque na prevenção e/ ou reabilitação (A16).

Ações realizadas que façam com que a pessoa não adoeça (A 20).

Os relatos expostos mostram o desconhecimento sobre o verdadeiro significado do tema promoção à saúde, havendo confusões entre os conceitos de promoção e prevenção. Diante disso, convém mencionar que há uma radical e, ao mesmo tempo, pequena diferença entre prevenção e promoção da saúde. Radical porque implica mudanças profundas na forma de articular e utilizar o conhecimento na formulação e operacionalização das práticas de saúde. Pequena diferença porque as práticas de promoção, tal como as de prevenção, fazem uso do conhecimento científico. As ações preventivas definem-se como intervenções orientadas a evitar o surgimento de doenças específicas, reduzindo sua incidência e prevalência nas populações. O modelo de saúde é meramente médico e dirige-se somente a grupos de risco da população(2).

O discurso apresentado pelos enfermeiros reflete o contexto da UTI, onde as práticas de cuidado concentram maior atenção às situações que exigem a utilização de equipamentos ou sobre o foco da doença e sua cura. A aplicabilidade da promoção da saúde mostra-se limitada, predominando o seguimento de normas e rotinas em suas estruturas. Com isso, excluem-se as possibilidades de atenção no intuito de promover a saúde em várias situações nas quais, por exemplo, a cura não se apresenta como alternativa única a ser alcançada ${ }^{(6-7)}$.

As concepções modernas de promoção da saúde estão voltadas para uma dimensão mais globalizante de saúde que considera as variáveis biológicas, psíquicas, sociais, culturais, ambientais. Referem-se a medidas voltadas não apenas para determinada doença, mas capazes de proporcionar saúde e bem-estar. Logo, tal dimensão requer trabalho interdisciplinar. Para tanto, incluir novos saberes significa compreender que o conhecimento hegemônico na clínica é indispensável à atenção em saúde, mas danoso quando utilizado isoladamente dos demais saberes ${ }^{(8)}$.

Ainda concernente ao conceito de promoção da saúde, o discurso apresentado pelos demais enfermeiros revelou que a maioria manifestou concepção mais abrangente do termo promoção da saúde, ou seja, uma visão holística.

Etimologicamente, a palavra holismo ou holístico vem do grego, holos, que significa todo, completo. A influência da visão holística nas práticas de saúde refere-se ao desenvolvimento de uma visão do ser humano inserido num contexto biopsicossocial, como um ser singular que traz consigo uma história de vida impregnada de valores culturais ${ }^{(9)}$.

Diante disso, no entendimento dos enfermeiros participantes da pesquisa, a promoção da saúde está atrelada à qualidade de vida do indivíduo, ao seu bem-estar e a medidas que proporcionam saúde, no seu aspecto físico, mental, social e emocional. Visualizaram o sujeito de forma integral, inserido em um contexto social e familiar, e enfatizaram a importância do trabalho interdisciplinar para que os problemas de saúde sejam compreendidos do ponto de vista de diferentes áreas do conhecimento, nas quais devem ser consideradas variáveis de ordem biológica, psicossocial, cultural e ambiental. A seguir, consta a transcrição de alguns discursos:
Percepção do enfermeiro sobre promoção da saúde na Unidade de Terapia Intensiva Aguiar ASC, Mariano MR, Almeida LS, Cardoso MVLML, Pagliuca LMF, Rebouças CBA 
É toda ação que promova o bem-estar físico e psíquico do paciente, profissional e família (A15).

(...) conjunto de estratégias interdisciplinares para atender os problemas de saúde do indivíduo, seja na dimensão biológica, psíquica ou ambiental (A29).

É toda ação que vise atender às necessidades do ser humano em todos os seus estados (mental, espiritual e físico) (A1).

São ações realizadas pelos profissionais de saúde e governantes para manter a integridade física, social e psicológica do ser humano (A5).

Medidas que orientam o cliente a ter uma melhor qualidade de vida (A19).

Nota-se nos relatos dos enfermeiros que, embora a promoção da saúde seja tema recorrente no cotidiano, apresenta-se como um conceito complexo e multifacetado.

As limitações inerentes à definição conceitual sobre promoção de saúde decorrem da própria dificuldade de se definir saúde, sobretudo em face das diferentes dimensões presentes no conceito: social, psicológica, econômica, espiritual, além da biomédica, mais tradicional. Sobressai, particularmente, o fato da saúde ser, antes de tudo, uma experiência individual. As formas como as pessoas percebem sua saúde e os meios como cuidam dela são tão diversos quanto as diferentes formas de significar e experimentar a vida(10).

Para a Organização Mundial da Saúde, a expressão promoção da saúde guarda relação com o holístico numa denotação mais política. Assim, as palavras revelam larga amplitude, contemplando diversas ações que envolvem todos os aspectos destinados a melhorar o status de saúde dos indivíduos. Promoção da saúde, portanto, compreende múltiplos aspectos, não constituindo uma atividade em si própria( ${ }^{(8,11)}$.

\section{Ações de promoção da saúde na unidade de terapia intensiva}

Esta categoria agrega as práticas de promoção da saúde que o enfermeiro evidencia no cuidado ao paciente internado em unidade de terapia intensiva. Dentre os enfermeiros participantes da pesquisa, 27 responderam desenvolver alguma estratégia de promoção da saúde, enquanto quatro responderam não desenvolver ou desenvolver raramente.

Acredita-se que as respostas dos enfermeiros, no que diz respeito à realização ou não de ações de promoção da saúde, sofreram influências tanto pela concepção que eles possuem acerca do conceito como pela visão relacionada à sua prática profissional.

Com base na análise dos discursos dos enfermeiros, quanto à realização de atividades de promoção da saúde no contexto da unidade de terapia intensiva, emergiram concepções variadas. Estas tiveram enfoque sobre os procedimentos, cuidados e controle de infecções, mas também sobre o usuário e seus familiares, além do ambiente de trabalho. Desse modo, emergiram as seguintes subcategorias: 2.1. Atenção voltada para o controle de infecções, tratamento e reabilitação; 2.2. Comunicação e apoio emocional ao paciente/família; 2.3. Promoção da saúde no ambiente da UTI; 2.4. Promoção da saúde com enfoque na educação em saúde.

\section{Atenção voltada para o controle de infecções, tratamento e reabilitação}

Na percepção dos enfermeiros, a efetivação de ações de promoção da saúde no cuidado ao paciente em UTI direciona-se predominantemente para a execução de procedimentos de cuidados com técnicas apropriadas para o tratamento das doenças e controle de infecções. Eis a transcrição dos discursos:

(...) administração de medicamentos e realização de procedimentos com técnica asséptica (A22).

Administração das dietas por sonda; incentivo à dieta via oral nos pacientes conscientes; realização de mudança de decúbito nos pacientes com mobilidade prejudicada (...) (A17).

(...) não deixar o paciente sem dieta ou com dieta inadequada; manter o paciente bem hidratado (A21).

Execução de procedimentos na assistência direta ao recém-nascido internado: aspiração traqueal, coleta de exames (...) (A5).

Lavagem das mãos, cuidados com a higienização, utilização de EPI's (A13).

(...) orientações aos pacientes e acompanhantes sobre medidas de prevenção de doenças (A4).

Em relação ao cuidado realizado em uma unidade de terapia intensiva e ao tipo de clientela atendida, as internações nesses centros são precedidas de complicações orgânicas, presentes e potenciais, as quais colocam em risco a vida do paciente. Esse fato tem contribuído para ser a assistência de enfermagem, nessas unidades, norteada pelo modelo biomédico. Predomina, assim, o cuidado voltado para os aspectos físicos da doença, como controle e manutenção das funções vitais ${ }^{(12)}$.

Portanto, como observado nos depoimentos dos enfermeiros, uma das concepções sobre desenvolvimento de atividades de promoção da saúde no cenário hospitalar ainda se aplica à realização de procedimentos, à prevenção de agravos, tratamento e cura de doenças.

Corrobora-se esta afirmação, pois a formação dos profissionais de saúde e dos enfermeiros direciona-se, em especial, aos aspectos biológicos e está centrada na prevenção, tratamento e ações curativas ${ }^{(6)}$. 
Conforme se percebe nos discursos apresentados, a preocupação com a execução de procedimentos e técnicas que levem à prevenção de doenças ou à adequada implementação de terapêuticas é confundida com o verdadeiro sentido da promoção da saúde no hospital. Embora as atitudes evidenciadas nos discursos dos enfermeiros sejam importantes e coerentes, é preciso ampliar a visão do cuidado como prática para promoção da saúde.

Na concepção de diversos autores, o papel do enfermeiro em UTI vai além da realização de procedimentos terapêuticos e do cuidado de sinais e sintomas físicos apresentados pelo cliente. Deve-se também identificar problemas, assistir o indivíduo em sua plenitude, com vistas a suprir as necessidades demonstradas, avaliando a assistência prestada e garantindo a eficácia da sua recuperação. Inclui-se, ainda, possibilitar o desenvolvimento da autonomia do usuário para habilitá-lo a participar do próprio cuidado e assumir um estilo de vida saudável, mesmo quando no ambiente hospitalar, preparando-se para a alta ${ }^{(6,8)}$.

\section{Comunicação e apoio emocional ao paciente/família}

Ainda que as ações citadas pelos enfermeiros concentrem-se sobremodo no modelo terapêutico e cura de doenças, dos discursos também emergiram práticas que preconizam a promoção da saúde em sentido ampliado, resgatando a humanização.

(...) conversar e tranquilizar os pacientes acordados. Procuro realizar estas atitudes na medida do possível (A21).

(...) dando apoio emocional aos pacientes, conversando com os mesmos, apesar de serem na maioria sedados e analgesiados (A23).

(...) comunicação com os pacientes conscientes, para que estes não desorientem (A17).

Orientar os pacientes quando conscientes, melhorando sua autoestima, estimulando sua saída da UTI com palavras consoladoras e amigas (A18).

Dialogar com pacientes acordados e interagir com os familiares (A15).

Orientar as famílias e os pacientes acordados quanto ao seu estado de saúde, condição clínica, práticas de vida saudáveis (...) (A24).

Assistência direta ao RN internado, orientações à família, apoio à mãe (...) (A5).

Como se depreende destes discursos, a comunicação com o paciente em unidade de terapia intensiva foi exposta pelos enfermeiros como um instrumento de promoção da saúde para o processo de cuidar. Vários estudos consideram a comunicação como uma importante ferramenta para a promoção e humanização da saúde (7-8,13-15). $^{\text {. }}$
Nas práticas de saúde no âmbito hospitalar, a comunicação potencializa a interação entre profissionais, pacientes e seus familiares. Dessa forma, torna as ações de cuidado mais humanizadas, reduzindo as dúvidas e angústias existentes no processo de hospitalização. Entretanto, o ato de se comunicar é um desafio para grande parte dos profissionais da enfermagem, especialmente aqueles que cuidam de pacientes em situações críticas de vida, tais como nas unidades de terapia intensiva.

A equipe de enfermagem numa UTI, envolvida pela rotina diária e complexa e pela prestação de serviço de alta tecnologia, muitas vezes, passa a apresentar menor interesse às relações pessoais. Esquece-se de tocar, conversar e ouvir o paciente ali à sua frente ${ }^{(11)}$.

Segundo evidenciado pelo discurso dos enfermeiros, a maioria referiu a comunicação com pacientes que estão conscientes. Embora nesse ambiente predominem pacientes com sua capacidade de expressão verbal prejudicada, em virtude, por exemplo, da intubação orotraqueal e da traqueostomia, é possível estabelecer a transmissão de mensagens através da comunicação não-verbal.

Poucos enfermeiros, contudo, mencionaram o uso da comunicação com pacientes sedados e analgesiados. Autores referem que o paciente em coma torna-se isolado no ambiente de internação pela incapacidade de se comunicar, constituindo-se, ainda, em um grande desafio para a equipe de enfermagem realizar o cuidado ${ }^{(15)}$.

Outro aspecto presente nas citações dos enfermeiros foi o vínculo com a família. A família tem se mostrado responsável por vários aspectos positivos relacionados à recuperação de seu familiar internado em uma UTI, satisfazendo muitas das suas necessidades e contribuindo com informações significativas a respeito do paciente, o que tem favorecido a tomada de decisões quanto à realização de procedimentos ${ }^{(7,15)}$.

\section{Promoção da saúde no ambiente da UTI}

Como mostram as falas, a preocupação com a interferência do ambiente da unidade de terapia intensiva no bem-estar do paciente acamado foi observada nos discursos dos enfermeiros como atitude de promoção da saúde. Em seus discursos eles ressaltam a importância da adoção de medidas que minimizem os estressores e beneficiem os clientes.

(...) procuro minimizar as fontes de estresse do paciente, na medida do possível (...) como reduzir os ruídos, tentar propiciar condições de sono e repouso tranquilo (A21).

Pratico a tranquilidade, a educação e o respeito no ambiente de trabalho (...) (A23).

(...) proporcionar ambiente tranquilo e seguro (A24).

(...) procuro fazer tudo para o bem-estar do paciente; alimentação, vestimenta, local de repouso adequado, etc (A25).
Percepção do enfermeiro sobre promoção da saúde na Unidade de Terapia Intensiva Aguiar ASC, Mariano MR, Almeida LS, Cardoso MVLML, Pagliuca LMF, Rebouças CBA 
Trabalhos publicados com ênfase em clientes internados em UTI apresentam depoimentos que apontam o barulho (de pessoas e aparelhos) como sendo o fator mais importante a ser controlado numa UTI. Dadas suas especificações técnicas de construção, a UTI possui uma área física restrita, o que permite que a pessoa ali internada enxergue ou perceba tudo ao seu redor. Além da presença de equipamentos, a dinâmica ininterrupta de trabalho da equipe, ruídos e alarmes constantes e monótonos, iluminação e aeração artificiais permanentes, falta de janelas para visualização do meio externo, etc., contribuem para alterar o emocional das pessoas nela tratadas. Assim, o ambiente físico pode desencadear distúrbios psicológicos, pela desorientação no tempo e no espaço, acrescidos da privação de sono motivada por ruídos constantes ${ }^{(11,16)}$.

Diante dessa situação, todos os aspectos passíveis de ser melhorados no intuito de minimizar os estressores, tornando o ambiente da UTI menos exaustivo e tenso, devem ser valorizados. Um deles diz respeito, por exemplo, à implementação de formas de relaxamento que promovam a harmonização do ambiente através da música, a diminuição dos ruídos responsáveis pela poluição sonora, a redução da luminosidade em alguns momentos e a manutenção da temperatura agradável. Estratégias que facilitam o contato, a interação e a dinâmica no contexto da UTI propiciam a promoção da saúde integral não só dos pacientes, como também dos profissionais.

\section{Promoção da saúde com enfoque na educação em saúde}

Alguns enfermeiros citaram em seus discursos o uso da educação em saúde como ferramenta para ações de promoção da saúde.

(...) orientações de educação em saúde para mães de recém-nascidos internados, sobre aleitamento materno, imunização (...) (A14).

(...) realizo orientações à família do paciente quanto à alta, procedimentos realizados (...) (A8).

Quando há pacientes conscientes, oriento-os acerca dos procedimentos cirúrgicos, por exemplo (A16).

(...) orientações às mães sobre cuidados com o recém-nascido; orientações sobre aleitamento materno (...) (A11).

(...) orientar as mães quanto aos cuidados que deve ter para prevenir infecções nos bebês (...) (A4).

Todas as atividades de promoção da saúde, incluindo a educação em saúde, precisam ser caracterizadas pelo empoderamento, isto é, ações que levem o indivíduo a sua autonomia e emancipação. Reconhecidamente, inúmeras práticas educativas desenvolvidas por enfermeiros enfocam a prevenção de doenças, sem, contudo, incorporar a compreensão dos fatores determinantes dos problemas de saúde ou as necessidades e saberes da população trabalhada ${ }^{(17-18)}$.
Segundo se observa nas falas, a referência à educação em saúde se fez majoritariamente pelos enfermeiros atuantes na unidade de terapia intensiva neonatal. $\mathrm{Na}$ concepção desses profissionais, a educação em saúde tem uma dimensão importante como estratégia de promoção da saúde, pois abre espaço para o profissional relacionar-se com a mãe, envolvendo-a no tratamento e incentivando-a a cuidar do seu bebê.

O processo educativo desenvolvido pelos enfermeiros na unidade neonatal é imprescindível, e as mães necessitam obter aprendizado no que se refere aos cuidados com o filho, pois o compartilhamento de experiências se reflete em ajuda mútua favorecendo assimilação e apreensão de conhecimentos ${ }^{(19)}$.

Ainda como observado nos discursos, poucos enfermeiros que trabalham em unidade de terapia intensiva adulto citaram a educação em saúde como atividade para a promoção da saúde devido aos fatores limitantes relacionados ao contexto da UTI, principalmente aqueles relacionados a própria condição clínica do paciente. Outros, quando se referiram a algum tipo de educação em saúde dentro da UTI, voltaram-se para explicações acerca de técnicas ou de procedimentos.

Como consta em estudos, a restrição do horário de visitas, o pouco contato com os familiares, o ambiente fechado e os fatores agravantes de saúde que levam os pacientes a se internarem na UTI foram apontados pelos enfermeiros como pontos negativos para a educação em saúde nesse setor ${ }^{(20)}$.

\section{Dificuldades para o desenvolvimento de ações de pro- moção da saúde}

Nesta categoria, apontaram-se algumas dificuldades mencionadas pelos enfermeiros quanto à realização de estratégias de promoção à saúde. Conforme os discursos dos enfermeiros, sete não encontram empecilhos para o desenvolvimento de ações de promoção da saúde no ambiente de trabalho onde atuam, ou seja, no contexto da unidade de terapia intensiva. Entretanto, a maioria dos enfermeiros reconhece algum tipo de dificuldade ou empecilho que limita a realização de atividades de promoção da saúde no ambiente da unidade terapia intensiva. Entre as dificuldades mais citadas estão: a sobrecarga de trabalho, a carência de material, a falta de empenho dos profissionais e o fato de muitos pacientes da UTI estarem sedados, o que dificulta a comunicação. Outro aspecto ressaltado pelos enfermeiros foi a falta de sensibilidade dos profissionais e a resistência a mudanças.

(...) existem dificuldades, como por exemplo, prestar uma assistência de qualidade (humanizada) devido ao grande número de tarefas (A25).

Ruídos de aparelhos; excesso de pessoas em um pequeno espaço. Manipulação excessiva nos pacientes; falta de algum tipo de terapia aos pacientes acordados que estão na UTI (A23). 
Falta de tempo; escassez de materiais; falta de empenho de alguns profissionais (A7).

(...) a maior parte dos pacientes encontram-se em estado grave, sedados, analgesiados, não sendo fácil estabelecer a comunicação (A26).

(...) a resistência a mudanças; velhos hábitos apegados (...) (A6).

Sensibilizar os profissionais para exercerem suas funções com envolvimento afetivo, de modo tal que não se tornem apenas tarefeiros mecânicos, mas com uma visão holística (A1).

(...) número de profissionais insuficientes para a demanda (...) (A5).

A cultura das pessoas não é de promoção e sim curativa (A20).

Estudo destacou como aspectos negativos a dificultar o relacionamento interpessoal do enfermeiro com o paciente em UTI: condutas mediadas por insensibilidade afetiva, o cuidado tecnicista e a ansiedade dos profissionais na rotina diária com pacientes graves. A grande demanda, os procedimentos e a agilidade na assistência também podem contribuir para uma impessoalidade na relação. Além de que, a preocupação com os aspectos administrativos do cuidado consome parte significativa das horas de trabalho do enfermeiro, favorecendo o distanciamento de suas metas ${ }^{(15)}$.

Um fator também citado pelos enfermeiros em seus discursos refere-se à sobrecarga de trabalho. Como apontam estudos, na maioria das vezes, os plantões transcorrem em um ambiente de agitação, e isto exige atenção e cuidado rigoroso de todos os integrantes da equipe ${ }^{(4,20)}$.

Outro aspecto mencionado refere-se à falta de recursos materiais. Ante a escassez de recursos materiais e humanos, os profissionais acabam fazendo o melhor que podem. Às vezes, é preciso improvisar. Tal iniciativa nem sempre traz benefícios ao paciente, e pode até culminar em prejuízo para a qualidade do cuidar ${ }^{(21)}$.

Nota-se nos discursos apresentados que dentre as dificuldades enfaticamente abordadas pelos enfermeiros que trabalham na UTI adulta menciona-se a seguinte: a maioria dos pacientes encontra-se sedado, e consequentemente com seu nível de consciência alterado. Desse modo, dificulta-se a comunicação.

Estudos apontam que a mecanização dos procedimentos técnicos, a falta de diálogo e a ausência de empatia acontecem, muitas vezes, porque o paciente em coma está inconsciente. Autores destacam que o comprometimento de algumas funções cerebrais e sensoriais não implica necessariamente na inexistência perceptual de expressões. Pesquisas mostram que pacientes em coma apresentam alterações fisiológicas quando ouvem uma música ou escutam uma voz familiar. Estes achados favorecem uma possibilidade de comunicação entre este e o meio ambiente ${ }^{(13,15)}$.

\section{CONCLUSÃO}

O conhecimento dos enfermeiros quanto ao conceito de promoção da saúde retratou distintas abordagens, variáveis desde uma visão biomédica de promoção da saúde, focada em medidas curativas e no processo de adoecimento, até uma concepção mais ampla, cuja finalidade é favorecer o bem-estar, transcendendo o enfoque estritamente biológico.

Apesar de as respostas relacionadas ao conceito sobre promoção da saúde apontar para a sua concepção mais abrangente, as estratégias apresentadas pelos enfermeiros direcionaram-se predominantemente para a realização de procedimentos de cuidados, para o tratamento das doenças e controle de infecções. Segundo observado, a maioria dos profissionais desconhece o verdadeiro significado do tema promoção da saúde, e muitas vezes confunde os conceitos de promoção e prevenção.

De modo geral as características particulares relacionadas ao contexto da UTI, como, por exemplo, as situações contínuas de emergência, a gravidade dos pacientes e a dinâmica acelerada do serviço, contribuem para comportamentos automatizados, nos quais o diálogo e a reflexão crítica não encontram espaço.

Ainda assim, alguns enfermeiros citaram atividades de promoção da saúde voltadas para aspectos amplos de atenção, abordando o usuário e seus familiares, como também o ambiente de trabalho. Desse modo, em seus discursos estiveram presentes ações de promoção da saúde direcionadas para a educação em saúde e para a comunicação e relacionamento enfermeiro-pacientefamília. Também se evidenciou a preocupação dos enfermeiros com a interferência do ambiente da UTI no bem-estar do paciente internado como atitude de promoção da saúde.

Dentre as dificuldades citadas pelos enfermeiros que limitam o desenvolvimento de ações de promoção da saúde no contexto da UTI estão: a sobrecarga de trabalho, a falta de sensibilidade dos profissionais, a resistência a mudanças e o estado clínico (nível de consciência alterado devido à sedação) da maioria dos pacientes que compromete a comunicação.

Aqui, alerta-se: algumas questões requerem contínua discussão. Ainda há muito a avançar para alcançar o desenvolvimento da promoção da saúde no contexto hospitalar, particularmente, no contexto da UTI, sobretudo porque as práticas de saúde instituídas no cuidado ao cliente são centradas hegemonicamente nos aspectos físicos da doença.

Apesar das características peculiares que permeiam o contexto da UTI e por esta ser um setor no qual se realiza um cuidado específico e complexo, destaca-se que
Percepção do enfermeiro sobre promoção da saúde na Unidade de Terapia Intensiva Aguiar ASC, Mariano MR, Almeida LS, Cardoso MVLML, Pagliuca LMF, Rebouças CBA 
não basta dominar o conhecimento junto à alta tecnologia existente nestas unidades. É necessário deter atenção particular no cliente em sua totalidade. Para isso, os profissionais devem ser atuantes, aplicar seus conhecimentos e habilidades, exercendo sua capacidade, além de técnica, política e social.

\section{REFERÊNCIAS}

1. Caponi S, Verdi M. Reflexões sobre a promoção da saúde numa perspectiva bioética. Texto Contexto Enferm. 2005;14(1):82-8.

2. Czeresnia D. O conceito de saúde e a diferença entre prevenção e promoção. In: Czeresnia D. Promoção da saúde: conceitos, reflexões, tendência. Rio de Janeiro: FIOCRUZ; 2003. p. 39-53.

3. Cunha RR, Pereira LS, Gonçalves ASR, Santos EKA, Radunz V, Heidemann ITSB. Promoção da saúde no contexto Paroara: possibilidade de cuidado de enfermagem. Texto Contexto Enferm. 2009;18(1):170-6.

4. Silva RCL, Porto IS, Figueiredo NMA. Reflexões acerca da assistência de enfermagem e o discurso de humanização em terapia intensiva. Esc Anna Nery Rev Enferm. 2008;12(1):156-9.

5. Bardin L. Análise de conteúdo. Lisboa: Edições 70; 1977.

6. Nunes JM, Martins AKL, Nóbrega MFB, Souza AMA, Fernandes AFC, Vieira NFC. Promoção da saúde no hospital sob a ótica do enfermeiro: estudo exploratório-descritivo. Online Bras J Nurs [Internet]. 2009 [citado 2009 dez. 18];18(3). Disponível em: http://www.objnursing.uff.br/index.php/nursing/article/ view/2568

7. Silva SG, Prochnow AG, Santos JLG, Guerra ST, Barrios SG. A comunicação entre a equipe de enfermagem e os familiares de pacientes em terapia intensiva: estudo qualitativo. Online Bras J Nurs [Internet]. 2009 [citado 2009 set. 2];8(2). Disponível em: http://www.objnursing.uff.br/index.php/nursing/ article/view/2317

8. Dias MAS, Vieira NFC. A comunicação como instrumento de promoção da saúde na clínica dialítica. Rev Bras Enferm. 2008;61(1):71-7.

9. Paula JAM, Paulino VCP. A necessidade de uma prática holística em saúde e a formação de professores dos cursos da área da saúde na Universidade Estadual de Goiás (UEG). Rev UFG [Internet]. 2005 [citado 2009 ago. 30];7(2). Disponível em: http://www.proec.ufg.br/revista_ufg/45anos/R-necessidade. html

10. Traverso-Yépez MA. Dilemas na promoção da saúde no Brasil: reflexões em torno da política nacional. Interface Comun Saúde Educ. 2007;11(22):223-38.
Diante disso, para que os serviços de saúde promovam a saúde, é necessário trabalho multidisciplinar e que os profissionais compreendam e ampliem sua visão de promoção à saúde, incluindo-se como autores críticos e participantes do processo de construção e reformulação deste sistema.

11. Silva GF, Sanches PG, Carvalho MDB. Refletindo sobre o cuidado de enfermagem em unidade de terapia intensiva. REME Rev Min Enferm. 2007;11(1):94-8.

12. Nascimento ERP, Trentini M. O cuidado de enfermagem na UTI: teoria humanística de Paterson e Zderad. Rev Latino Am Enferm. 2004;12(2):250-7

13. Zinn GR, Silva MJP, Telles SCR. Comunicar-se com o paciente sedado: vivência de quem cuida. Rev Latino Am Enferm. 2003;11(3):326-32.

14. Toralles-Pereira ML, Sardenberg T, Mendes HWB, Oliveira RA. Comunicação em saúde: algumas reflexões a partir da percepção de pacientes acamados em uma enfermaria. Ciênc Saúde Coletiva. 2004;9(4):1013-22.

15. Siqueira $A B$, Filipina R, Posso MBS, Fiorano AMM, Gonçalves $S A$. Relacionamento enfermeiro, paciente e família: fatores comportamentais associados à qualidade da assistência. Arq Med ABC. 2006;31(2):73-7.

16. Faquinello P, Dióz M. A UTI na ótica de pacientes. REME Rev Min Enferm. 2007;11(1):41-7.

17. Acioli S. A prática educativa como expressão do cuidado em Saúde Pública. Rev Bras Enferm. 2008;61(1):117-21.

18. Becker D, Edmundo K, Nunes NR, Bonatto D, Souza R. Empowerment e avaliação participativa em um programa de desenvolvimento local e promoção da saúde. Ciênc Saúde Coletiva. 2004;9(3):655-67.

19. Lélis ALP, Machado MFAS, Cardoso MVLML. Educação em saúde e a prática de Enfermagem ao recém-nascido prematuro. Rev RENE. 2009;10(4):60-9.

20. Preto VA, Pedrao LJ. Stress among nurses who work at the Intensive Care Unit. Rev Esc Enferm USP [Internet]. 2009 [cited 2010 May 12];43(4):841-8. Available from: http://www. scielo.br/pdf/reeusp/v43n4/en_a15v43n4.pdf

21. Leite MA, Vila VSC. Dificuldades vivenciadas pela equipe multiprofissional na unidade de terapia intensiva. Rev Latino Am Enferm. 2005;13(2):145-50. 